\title{
The Contribution of Social Media to Body Dissatisfaction, Eating Disorder Symptoms, and Anabolic Steroid Use Among Sexual Minority Men
}

\author{
Scott Griffiths, PhD, Stuart B. Murray, PhD, Isabel Krug, PhD, and Siân A. McLean, $\mathrm{PhD}^{3,4}$
}

\begin{abstract}
Social media has been associated with body dissatisfaction and eating disorder symptoms among young women and adolescent girls. However, despite notable evidence of susceptibility to body image pressures, it remains unknown whether these associations generalize to sexual minority men. A nationwide sample of 2,733 sexual minority men completed an online survey advertised to Australian and New Zealand users of a popular dating app. Participants answered questions about how frequently they used 11 different social media platforms in addition to questions about their dating app use, body image, eating disorder symptoms, and anabolic steroids. Facebook, Youtube, Instagram, and Snapchat were the most frequently used social media platforms. A pattern of small-sized and positive associations emerged between social media use and body dissatisfaction, eating disorder symptoms, and thoughts about using anabolic steroids. Facebook, Instagram, and Snapchat evidenced the strongest associations. The associations of social media use with both muscularity dissatisfaction and eating disorder symptoms were stronger for image-centric social media platforms (e.g., Instagram) than nonimagecentric platforms (e.g., Wordpress); no differences were observed for body fat dissatisfaction, height dissatisfaction, or thoughts about using anabolic steroids. Previously documented associations of social media use with body dissatisfaction and related variables among women and girls appear to generalize to sexual minority men. Social media platforms that more centrally involve imagery may be of greater concern than nonimagecentric platforms. Additional research with sexual minority men is needed to elucidate the distinctions between adaptive and maladaptive social media use in the context of body dissatisfaction, eating disorders, and anabolic steroid use.
\end{abstract}

Keywords: social media, body image, eating disorders, anabolic steroids, men, sexual minority men

\section{Introduction}

S OCIAL MEDIA IS a nontraditional form of media that has become increasingly popular in Australia ${ }^{1}$ and countries worldwide. ${ }^{2}$ According to the 2017 Sensis Social Media Report, ${ }^{1} 79$ percent of Australians use social media, including 99 percent of 18-29-year olds. Relatedly, researchers have found evidence to suggest that exposure to social media may cause body dissatisfaction and eating disorders through various theoretical mechanisms, including physical appearance comparisons and self-objectification. ${ }^{3-11}$ In this context, maladaptive social media use would include persistent user engagement in these mechanisms of body dissatisfaction and eating disorder development. For example, a user might frequently access, and compare themselves with, objectifying social media imagery containing depictions of very thin bodies or lean and muscular bodies; imagery that some researchers have termed "thinspiration" and "fitspiration," respectively. ${ }^{12,13}$ The effects of social media may be more potent than those of traditional forms of media (e.g., television and magazines) due to its continual availability (e.g., on smartphones), ${ }^{14}$ heightened salience from interacting with a

\footnotetext{
${ }^{1}$ Melbourne School of Psychological Sciences, University of Melbourne, Melbourne, Australia.

${ }^{2}$ School of Medicine, University of California San Francisco, San Francisco, California.

${ }^{3}$ College of Health and Biomedicine, Victoria University, Melbourne, Australia.

${ }^{4}$ School of Psychology and Public Health, La Trobe University, Melbourne, Australia.
}

(C) Scott Griffiths et al. 2018; Published by Mary Ann Liebert, Inc. This is an Open Access article distributed under the terms of the Creative Commons Attribution License, which permits unrestricted use, distribution, and reproduction in any medium, provided the original work is properly cited. 
similar peer group (similar in age, location, interests, etc.), and in-built real-time evaluative components (e.g., "liking", a photo). ${ }^{15}$

Fardouly and Vartanian, ${ }^{16}$ in their review of social media and body image research, noted that the field is predominated by studies of young women and adolescent girls. By contrast, sexual minority men have received scant attention, despite evidence that they constitute a highly vulnerable subgroup. For example, Frederick and Essayli ${ }^{17}$ compared some 112,000 heterosexual and 4,400 sexual minority men on different aspects of body image, and found, among sexual minority men, greater muscularity dissatisfaction, greater self-objectification, and more frequent appearance-based social comparisons, with small-to-medium effect sizes. Notably, both self-objectification and appearance-based social comparisons are operative mechanisms in theories of how social media exposure causes body dissatisfaction and eating disorders. ${ }^{3-11}$ Furthermore, sexual minority men experience elevated rates of psychiatric disorders for which body dissatisfaction is a central component, including eating disorders ${ }^{18,19}$ and body dysmorphic disorder. ${ }^{20,21}$ Thus, there is a need to examine, in sexual minority men, potential risk factors for body dissatisfaction, inclusive of social media use.

Fardouly and Vartanian ${ }^{16}$ further noted in their review that most studies of social media have focused on Facebook despite the growing popularity of alternative platforms, including Twitter, Instagram, and Snapchat. The authors note that several of these alternative platforms, including Instagram, are more image focused than Facebook, and thus, offer greater opportunity for users to engage in the processes theorized to lead to body dissatisfaction, including, for example, physical appearance comparisons. ${ }^{16}$ Furthermore, there is evidence that the increasing popularity of social media is disproportionately driven by image-centric social media platforms, that is, platforms that contain a high proportion of content that is image based, particularly images of people, relative to text or other content. ${ }^{1,22}$ For example, Snapchat, an image-centric social media platform, doubled in popularity among Australians from 2016 to 2017 . $^{1}$

Dating applications ("apps" for short) are a form of social media used by individuals seeking sexual relationships ${ }^{23,24}$ that, to date, have received little attention in the body image literature. The rationale for examining dating apps in relation to body dissatisfaction is compelling. Dating apps, which include, for example, Tinder and Grindr, are often heavily or exclusively image-focused, and their use involves real-time user feedback that can be either positive or negative. Limited research has examined the associations of dating apps with body dissatisfaction and related variables. Strubel and Petrie, ${ }^{15}$ in a study of 100 Tinder users and 847 nonusers (20.4 percent men), found that Tinder users reported greater body dissatisfaction and more frequent physical appearance self-comparisons, and the strength of these associations did not differ by sex. Hitherto, the use of dating apps has not been explored among sexual minority men, despite evidence that the use of dating apps is extremely common within the sexual minority male community. ${ }^{25}$

It is important to note that men's body image concerns are qualitatively different to women's body image concerns. ${ }^{26-29}$ For example, men, and sexual minority men in particular, desire an overtly muscular body. ${ }^{17}$ Thus, it is prudent to examine both muscularity and body fat dissatisfaction as criterion variables in studies of men's body image, since overt muscularity is achieved by combined means of developed musculature and low body fat. An additionally important criterion variable is anabolic steroids. Anabolic steroids are used predominantly by men ${ }^{30}$ with muscularity-focused body dissatisfaction $^{31}$ and their use is considerably more common among sexual minority men than among heterosexual men. ${ }^{32,33}$ Finally, it is important to consider both height dissatisfaction and eating disorder symptoms as criterion variables. Regarding the former, height dissatisfaction is a dimension of body image that is notably relevant to men, ${ }^{34}$ including sexual minority men. ${ }^{35}$ Regarding the latter, eating disorders are a syndrome of psychopathology for which body dissatisfaction is a key component and are more common among sexual minority men relative to their heterosexual counterparts. ${ }^{19,36}$

Thus, this study aimed to determine whether, and to what extent, the frequency of use of various social media platforms is associated with body dissatisfaction, eating disorder symptoms, and thoughts about using anabolic steroids, among sexual minority men. Two hypotheses were made: first, that more frequent use of social media would be associated with greater body dissatisfaction, eating disorder symptoms, and more frequent thoughts about using anabolic steroids, and second, that these relationships would be stronger for more image-centric social media platforms (e.g., Instagram) than less image-centric platforms (e.g., LinkedIn).

\section{Method}

\section{Procedure}

Advertisements that solicited volunteers for a study of body image and body change behaviors were broadcast on a popular geosocial dating app used exclusively by sexual minority men. The text-based advertisements appeared nationwide in Australia and New Zealand on 4 nonconsecutive days in April and May 2017. Clicking the link in the advertisement directed potential participants to the online survey. Median survey completion time was 11 minutes. The study protocol was reviewed and approved by the University of Melbourne.

\section{Measures}

Demographics and anthropometrics. Participants provided their age, gender, sexual orientation, relationship status, and cultural background. Sexual orientation was assessed using a 5-point Kinsey-like scale ${ }^{37}$; response options ranged from "exclusively gay" to "exclusively heterosexual" with "bisexual" as the midpoint. Cultural background was assessed using response options reproduced from the Australian Standard Classification of Cultural and Ethnic Groups. ${ }^{38}$ Relationship status was assessed using the following response options: "single," "in a casual relationship (e.g., 'friends with benefits')," "in a serious relationship," "in a civil union, civil, or domestic partnership," or "married."

Social media and dating use. We asked participants to rate how frequently they used various forms of social media, namely, Facebook, Youtube, Instagram, Snapchat, Tumblr, Twitter, LinkedIn, Twitter, Wordpress, Pinterest, Flickr, and Blogspot. These social media platforms were chosen after consultation with members of the target population (i.e., sexual minority Australian men) and after an analysis of a recent report on Australians' social media habits. ${ }^{1}$ We also asked participants to rate how frequently they used various 
forms of dating apps. We were unable to name individual dating apps due to recruitment through advertising on a specific dating app; thus, we asked one general question: "How frequently do you use dating/hook-up apps?" Response options for the questions about social media and dating apps were "never," "rarely," "sometimes," "often," "very often," and "all the time." These response options correspond to scores of 1 through 6 , with higher scores indicating more frequent usage.

While acknowledging that visual content and images may technically be portrayed on all social media platforms of interest, we broadly distinguished between image-centric and nonimage-centric social media platforms by (a) examining the amount and prominence of imagery on each platform and by (b) consulting previous research on social media that distinguishes image-centric social media from nonimagecentric social media (e.g., Refs. ${ }^{1,22}$ ). Thus, the social media platforms were categorized as follows: image-centric (Facebook, Snapchat, Flickr, Instagram, Pinterest, Youtube, and Tumblr) and nonimage-centric (Twitter, LinkedIn, Wordpress, and Blogspot).

Body dissatisfaction. The Male Body Attitudes ScaleRevised (MBAS-R) ${ }^{39}$ was used to measure dissatisfaction with body fat (five items), muscularity (seven items), and height (three items). Items on the MBAS-R ask respondents to indicate how frequently they endorse various statements (e.g., "I think my arms should be more muscular'") using a 5-point response scale ranging from "never" to "always.", These response options correspond to scores of 1 through 5, with higher scores indicating greater body dissatisfaction. In previous studies, the MBAS-R has demonstrated sound internal reliability (Cronbach's $\alpha \mathrm{S}=0.88-0.91$ across the three subscales) ${ }^{39}$ and superior psychometric properties relative to its predecessor, the MBAS. ${ }^{40}$ In this study, Cronbach's alpha values for the body fat, muscularity, and height subscales were $0.92,0.88$, and 0.76 , respectively.

Eating disorder symptoms. The Eating Disorders Examination Questionnaire Short (EDE-QS) ${ }^{41}$ was used to measure core symptoms of eating disorders. The 12 items on the EDE-QS ask respondents to indicate the frequency with which they have experienced various eating disorder symptoms during the past week (e.g., "On how many of the past 7 days have you tried to control your weight or shape by making yourself sick or taking laxatives?"') using a 4-point response scale ranging from "0 days" to "6-7 days." These response options correspond to scores of 0 through 3 , with higher scores indicating more severe eating disorder symptoms. In previous studies, the EDE-QS has demonstrated sound internal reliability (Cronbach's $\alpha=0.91$ ) and superior psychometric properties relative to its predecessor, the EDEQ. ${ }^{42}$ In this study, Cronbach's alpha was 0.88 .

Thoughts about using anabolic steroids. Verbatim, we asked participants "how frequently do you think about using anabolic steroids?" In designing this question, we incorporated recommendations made by Kanayama et al. ${ }^{43}$ First, we used the term "anabolic steroids" rather than the less specific term "steroids," and second, we provided participants with the names of three anabolic steroids commonly used in Australia and New Zealand: testosterone enanthate, dianabol, and clenbuterol. ${ }^{44}$ Response options ranged from "never" to "always" and corresponded to scores of 1 through 6, with higher scores indicating more frequent thoughts about using anabolic steroids.

\section{Participants}

The survey was accessed by 3,756 potential participants. Of these, 397 did not provide consent and 142 provided

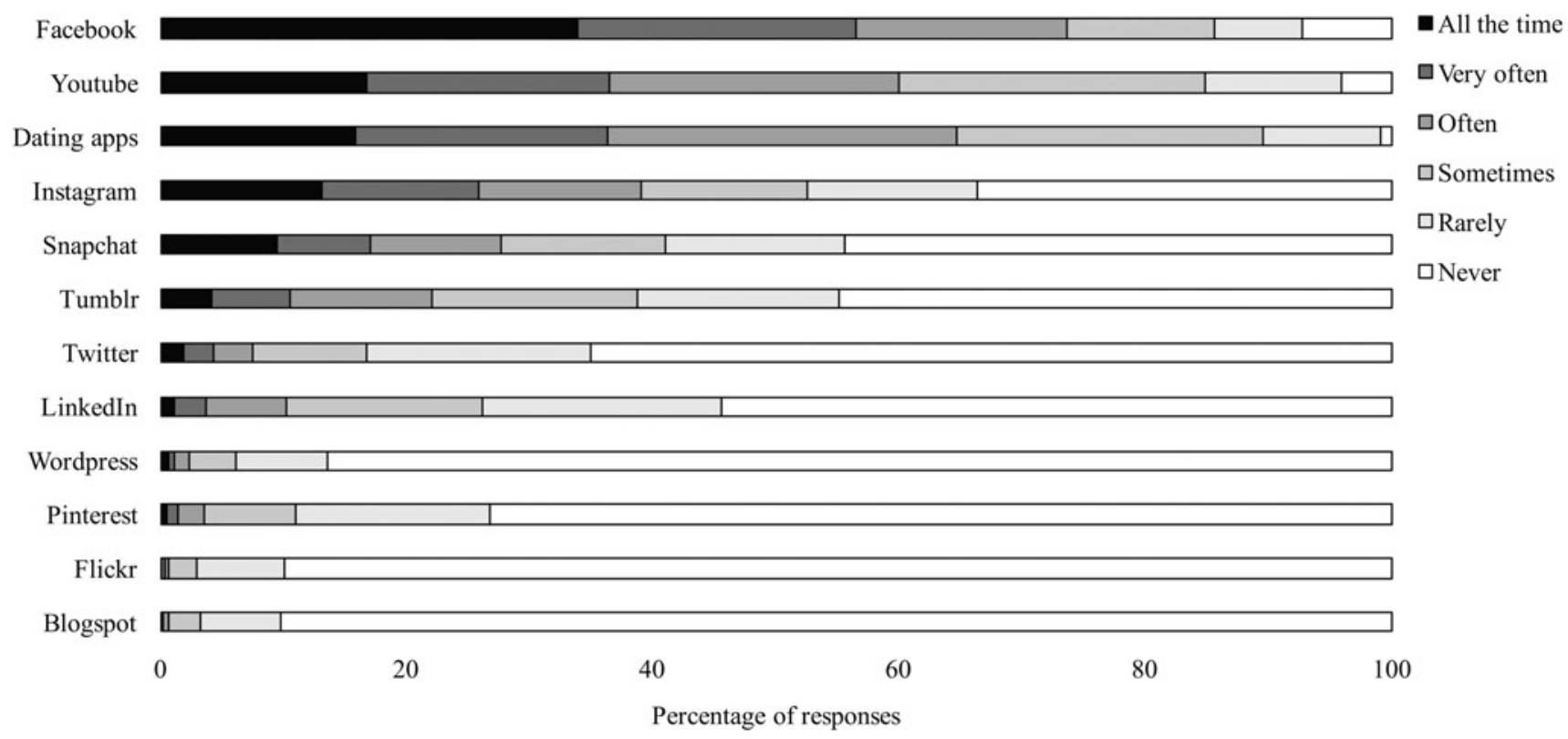

FIG. 1. The proportion of participants who selected each response option when asked to rate how frequently they use various social media platforms and dating apps. Available response options were "all the time," "very often," "often," "sometimes," "rarely," and "never." The social media platforms and dating apps on the Y-axis are sorted in descending order based on the proportion of participants who selected "all the time" as their response. 


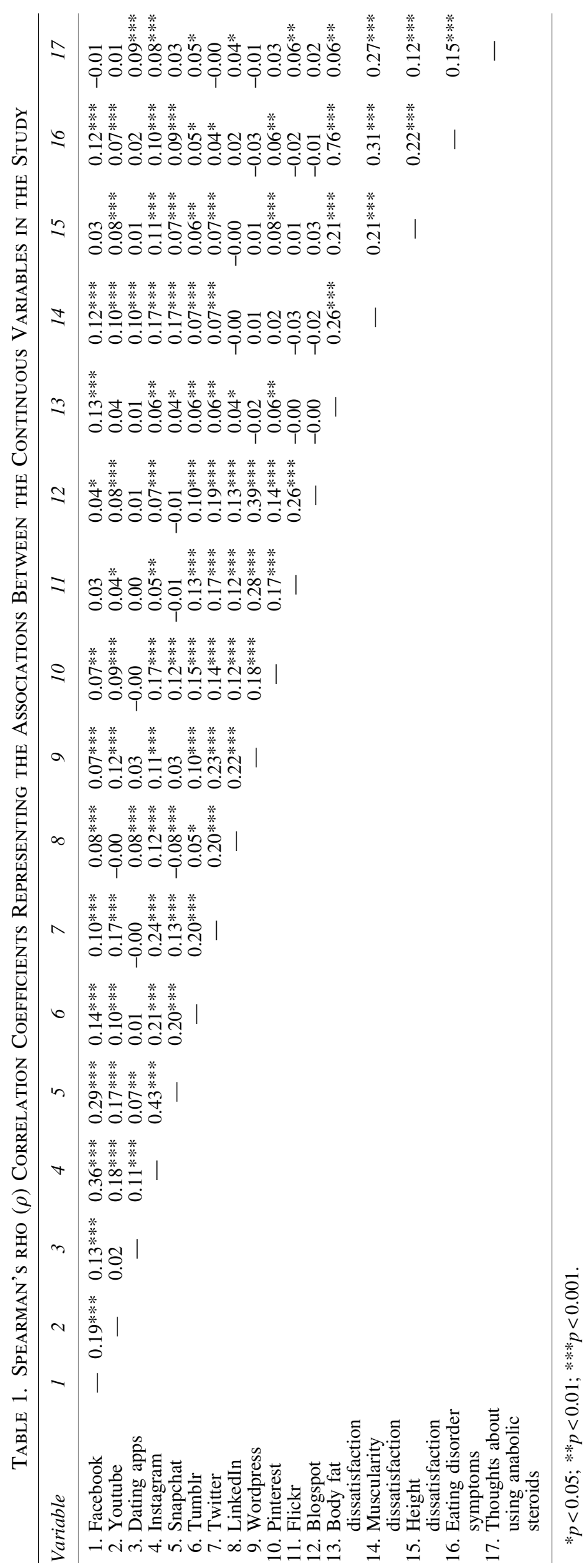


consent but did not provide any data. Of the remaining 3,217 participants, 452 were excluded because of providing high amounts of missing data ( $>30$ percent missing), 5 were excluded because of providing a systematic pattern of implausible data, 25 were excluded because of being younger than 18 years of age, and 2 were excluded because of specifying their gender as female. After data exclusions, the sample comprised 2,733 participants.

Participants almost exclusively self-identified as male gender ( 99.1 percent) ( 0.3 percent identified as transgender and 0.6 percent identified as "other"). All participants specified minority sexual orientations (68.4 percent exclusively gay/homosexual, 21.4 percent mostly gay/homosexual, 8.4 percent bisexual, 1.1 percent mostly straight/heterosexual, 0.7 percent "'other") . Ages ranged from 18 through 78 years $(M=33.93, S D=11.94)$. All participants resided in either Australia (78.7 percent) or New Zealand (21.3 percent). Cultural backgrounds were predominantly Australian (50.6 percent) and New Zealand (15.4 percent), followed by Northwest European (8.0 percent) and Southeast Asian (6.4 percent). Participants were predominantly single (66.0 percent), followed by "in a serious relationship" (13.9 percent), "in a casual relationship" ( 9.5 percent), in a civil union, civil partnership, or domestic partnership (7.9 percent), married (1.1 percent), and "other" (1.1 percent).

\section{Statistical analyses}

First, we visualized how frequently participants used social media and dating apps. Second, we conducted Spearman's correlational analyses to examine bivariate associations between the continuous variables in the study. Given the large sample size, only correlations of at least small effect size $(\rho=0.10)$ were deemed worthy of interpretation. Third, we visualized the correlation coefficients representing the associations between frequency of use of various social media platforms and dating apps with the criterion variables. Fourth, we calculated descriptive statistics for the aforementioned correlation coefficients as a function of whether they were image centric or nonimage centric and compared the strength of the mean correlation coefficients for image-centric and nonimage-centric social media platforms using a Fishers' $r$-to- $Z$ transformation. Fifth, we conducted a series of generalized linear regression models predicting our criterion variables. Two of these models were gamma-corrected because of positive skew in the criterion variable data distributions (height dissatisfaction and thoughts about using anabolic steroids).

\section{Results}

Figure 1 shows that Facebook was the most frequently used social media platform, with nearly 40 percent of participants reporting that they use Facebook "all the time." Table 1 shows a pattern of positive intercorrelations among the social media variables, indicating that frequent users of one social media service were likely to be frequent users of additional services. Figure 2 shows Spearman's rho correlation coefficients representing the associations between frequency of use of various social media platforms and dating apps with the criterion variables. Overall, there was a pattern of small-sized positive relationships of social media and dating app use with body dissatisfaction, eating disorder symptoms, and thoughts about using anabolic steroids. Table 2 shows that, on average, the associations of social media use with muscularity dissatisfaction and eating disorder symptoms were stronger for image-centric social media platforms than for nonimage-centric social media

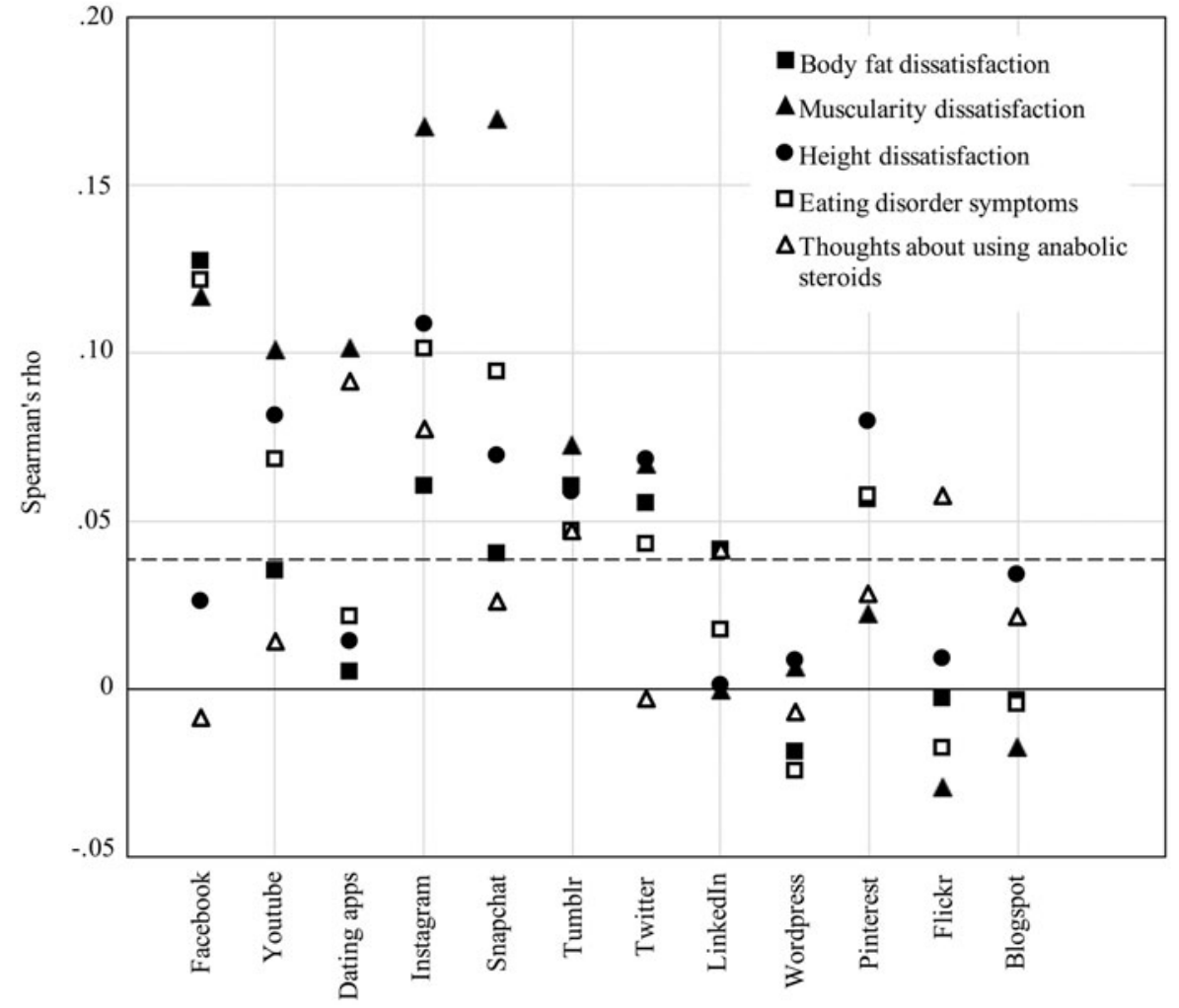

FIG. 2. Scatterplot of Spearman's rho $(\rho)$ correlation coefficients representing the associations between frequency of use of various social media platforms and dating apps with body fat dissatisfaction, muscularity dissatisfaction, height dissatisfaction, eating disorder symptoms, and thoughts about using anabolic steroids. The dashed horizontal line at $\rho=0.04$ represents the conventional significance threshold of $\alpha=0.05$. Correlation coefficients above and below this line were significant and nonsignificant, respectively. 
Table 2. Descriptive Statistics and Fisher's $R$-To-Z Significance Tests Examining Whether the Spearman Correlations Representing the Associations of Social Media Use with the Criterion Variables Differ as a Function of Whether the Social Media Platform Being Used Is Image Centric Versus Nonimage Centric

\begin{tabular}{|c|c|c|c|c|c|c|c|c|c|}
\hline \multirow[b]{2}{*}{ Variable } & \multicolumn{3}{|c|}{$\begin{array}{c}\text { Image-centric } \\
\text { social media platforms }\end{array}$} & \multicolumn{3}{|c|}{$\begin{array}{l}\text { Nonimage centric } \\
\text { social media platforms }\end{array}$} & \multicolumn{3}{|c|}{$\begin{array}{l}\text { Fisher's r-to-Z } \\
\text { significance test }\end{array}$} \\
\hline & Range & Mean & SD & Range & Mean & SD & Difference & $\mathrm{Z}$ & $\mathrm{p}$ \\
\hline Body fat dissatisfaction & -0.003 to 0.127 & 0.054 & 0.039 & -0.019 to 0.055 & 0.019 & 0.035 & 0.035 & 1.28 & 0.201 \\
\hline Muscularity dissatisfaction & -0.030 to 0.169 & 0.075 & 0.079 & -0.018 to 0.066 & 0.013 & 0.037 & 0.062 & 2.26 & $\mathbf{0 . 0 2 4}$ \\
\hline Height dissatisfaction & 0.009 to 0.108 & 0.061 & 0.034 & 0.001 to 0.068 & 0.028 & 0.030 & 0.034 & 1.20 & 0.230 \\
\hline Eating disorder symptoms & -0.018 to 0.121 & 0.067 & 0.046 & -0.025 to 0.043 & 0.007 & 0.029 & 0.070 & 2.19 & $\mathbf{0 . 0 2 9}$ \\
\hline $\begin{array}{l}\text { Thoughts about using } \\
\text { anabolic steroids }\end{array}$ & -0.009 to 0.077 & 0.034 & 0.029 & -0.006 to 0.041 & 0.013 & 0.022 & 0.021 & 0.76 & 0.447 \\
\hline
\end{tabular}

Bold denotes a significant difference at $\alpha=0.05$.

$S D$, standard deviation.

platforms. No significant differences were observed for body fat dissatisfaction, height dissatisfaction, or thoughts about using anabolic steroids. Table 3 shows that the generalized linear regression models largely supported the aforementioned findings; the most consistent (although weak) associations were observed for image-centric social media platforms. All models were significant (likelihood ratio $\left.\chi^{2} \mathrm{~s}=51.84-134.35, p s<0.001\right)$.

\section{Discussion}

In support of our first hypothesis, our findings showed that higher frequency of use of social media platforms, particularly Facebook, Instagram, and Snapchat, corresponded to greater body image concerns and eating disorder symptoms. In particular, muscularity dissatisfaction was shown to have positive associations across a number of social media platforms, whereas other indicators of body image concerns, body fat and height dissatisfaction, and eating disorder symptoms were associated with social media use on only one or two of the platforms. The study findings also partially supported the second hypothesis: that body image and related variables would be more strongly associated with use of image-centric than nonimage-centric social media platforms. Specifically, the associations between both muscularity dissatisfaction and eating disorder symptoms with frequency of social media use were significantly stronger for imagecentric than for nonimage centric social media platforms. Contrary to our expectations, the strength of the association between the two categories of social media platforms did not differ for body fat dissatisfaction, height dissatisfaction, or thoughts about anabolic steroid use.

This study is the first to demonstrate that greater body dissatisfaction and eating disorder symptoms are associated with greater frequency of social media use, particularly on imagecentric platforms, among sexual minority men. Although to

Table 3. Summary Statistics from the Generalized Linear Regression Models Predicting Body Fat Dissatisfaction, Muscularity Dissatisfaction, Height Dissatisfaction, Eating Disorder Symptoms, and Frequency of Thoughts About Using Anabolic Steroids

\begin{tabular}{|c|c|c|c|c|c|c|c|c|c|c|c|c|c|c|c|}
\hline \multirow[b]{4}{*}{ Independent variable } & \multicolumn{15}{|c|}{ Dependent variable } \\
\hline & \multicolumn{3}{|c|}{$\begin{array}{c}\text { Body fat } \\
\text { dissatisfaction }\end{array}$} & \multicolumn{3}{|c|}{$\begin{array}{c}\text { Muscularity } \\
\text { dissatisfaction }\end{array}$} & \multicolumn{3}{|c|}{$\begin{array}{c}\text { Height } \\
\text { dissatisfaction }\end{array}$} & \multicolumn{3}{|c|}{$\begin{array}{c}\text { Eating } \\
\text { disorder } \\
\text { symptoms }\end{array}$} & \multicolumn{3}{|c|}{$\begin{array}{l}\text { Thoughts about } \\
\text { using anabolic } \\
\text { steroids }\end{array}$} \\
\hline & \multicolumn{3}{|c|}{ Wald } & \multicolumn{3}{|c|}{ Wald } & \multicolumn{3}{|c|}{ Wald } & \multicolumn{3}{|c|}{ Wald } & \multicolumn{3}{|c|}{ Wald } \\
\hline & $\beta$ & $\chi^{2}$ & $\mathrm{p}$ & $\beta$ & $\chi^{2}$ & $\mathrm{p}$ & $\beta$ & & $\mathrm{p}$ & $\beta$ & 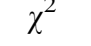 & $\mathrm{p}$ & $\beta$ & $\chi^{2}$ & $\mathrm{p}$ \\
\hline Face & 0.08 & 23.15 & $<0.001$ & 0.02 & 2.62 & 0.106 & -0.004 & 0.53 & 0.469 & 0.03 & 11.6 & 0.001 & -0.02 & 11.55 & 0.001 \\
\hline Youtube & -0.01 & 0.01 & 0.957 & 0.04 & 8.96 & 0.003 & 0.019 & 8.71 & 0.003 & 0.01 & 2.45 & 0.119 & 0.01 & 0.82 & 0.366 \\
\hline Dating apps & -0.01 & 0.41 & 0.522 & 0.04 & 10.28 & 0.001 & 0.007 & 0.96 & 0.328 & 0.01 & 0.01 & 0.917 & 0.04 & 28.39 & $<0.001$ \\
\hline Instagram & -0.01 & 0.01 & 0.909 & 0.05 & 18.47 & $<0.001$ & 0.018 & 9.94 & 0.002 & 0.01 & 1.50 & 0.221 & 0.03 & 20.73 & $<0.001$ \\
\hline Snapchat & -0.01 & 0.01 & 0.938 & 0.04 & 15.16 & $<0.001$ & 0.005 & 0.89 & 0.346 & 0.02 & 8.12 & 0.004 & -0.01 & 0.10 & 0.758 \\
\hline Tumblr & 0.04 & 6.28 & 0.012 & 0.02 & 2.15 & 0.143 & 0.009 & 2.27 & 0.132 & 0.01 & 0.81 & 0.369 & 0.01 & 2.20 & 0.138 \\
\hline Twitter & 0.02 & 1.02 & 0.312 & 0.02 & 1.30 & 0.255 & 0.008 & 0.93 & 0.335 & 0.01 & 0.12 & 0.731 & -0.02 & 6.23 & 0.013 \\
\hline LinkedIn & 0.04 & 3.02 & 0.082 & -0.01 & 0.06 & 0.806 & -0.001 & 0.01 & 0.922 & 0.01 & 0.76 & 0.384 & 0.01 & 0.65 & 0.421 \\
\hline Wordpress & -0.05 & 2.16 & 0.142 & -0.01 & 0.02 & 0.886 & -0.032 & 5.61 & 0.018 & -0.03 & 2.55 & 0.110 & -0.02 & 2.16 & 0.142 \\
\hline Pinterest & 0.05 & 3.42 & 0.064 & -0.02 & 0.54 & 0.464 & 0.019 & 3.24 & 0.072 & 0.03 & 2.92 & 0.088 & 0.01 & 1.39 & 0.238 \\
\hline Flickr & -0.05 & 1.12 & 0.290 & -0.07 & 3.28 & 0.070 & -0.002 & 0.01 & 0.937 & -0.03 & 1.31 & 0.253 & 0.04 & 2.57 & 0.109 \\
\hline Blogspot & -0.03 & 0.34 & 0.561 & -0.03 & 0.76 & 0.384 & 0.045 & 5.10 & 0.024 & -0.01 & 0.07 & 0.792 & 0.02 & 0.68 & 0.410 \\
\hline
\end{tabular}

Bold denotes statistical significance at $\alpha=0.05$. 
date, these relationships have not been investigated in sexual minority men, the findings of this study are consistent with previous research in samples of heterosexual men. For example, among college-aged men, greater time spent using Facebook, an image-centric platform, was found to be associated with self-objectification and body shame, both of which are indicators of body dissatisfaction. ${ }^{45,46}$ It is important to note that the effect sizes reported in this study for the relationships between social media use and body image concerns were small, even for image-centric platforms. However, in this study, assessment of use of these platforms did not distinguish between appearance-related and nonappearance-related engagement, for example, following fitspiration versus travel accounts on Instagram. Thus, effects may have been somewhat diluted.

Findings from this study, that the associations between social media use and muscularity dissatisfaction and eating disorder symptoms were stronger for image-centric than for nonimage-centric social media platforms, are consistent with previous research conducted with adolescent girls in which body dissatisfaction and disordered eating were associated with appearance-related activities on social media. ${ }^{4,48} \mathrm{Al}-$ though these two populations differ in a number of respects, this emerging body of research suggests that the relationship between image-centric social media use and body image is consistent across different sectors of the community. Thus, focus on appearance and image presented in social media may invoke similar processes in sexual minority men as for adolescent girls whereby engagement with these environments prompts internalization of appearance ideals and appearance comparison, highlighting the discrepancy between one's current appearance and ideals in social media, leading to body dissatisfaction. ${ }^{7,47}$

In line with expectations, the use of dating apps was associated with body image variables. In multivariate analyses, dating app use was associated with muscularity dissatisfaction and thoughts about anabolic steroids. In univariate analyses, however, the size of these associations was small to negligible. Although dating apps are highly image centric, it is possible that the associations of dating app use with body dissatisfaction and related variables are moderated by the relative amounts of positive and negative feedback received from others. In relation to thoughts about anabolic steroids, the weak associations with social media use may reflect that anabolic steroid use is a significantly more distal outcome than muscularity dissatisfaction or related forms of body dissatisfaction, thus attenuating the strength of these associations.

Limitations of the study are noted. Frequency of social media use was assessed with descriptive terms (never through to always) rather than specific periods of time that may have been interpreted inconsistently by participants. Furthermore, we were unable to list the specific names of dating apps, which may have led to an underestimation of dating app use if participants did not recall their use without prompting of specific apps. Finally, we are cognizant of the fact that the dichotomization of social media platforms as image centric versus nonimage centric is, to at least some extent, subjective, and that variability in the amount and prominence of imagery likely reflect both the developers' intended functions for the platform and the ways in which users interact with the platform. Future research is needed to elucidate the nature of maladaptive social media use in the context of body dissatisfaction and eating disorders among sexual minority and heterosexual men.

\section{Author Disclosure Statement}

No competing financial interests exist.

\section{References}

1. Sensis. (2017) Sensis social media report 2017. Melbourne, Australia: Sensis.

2. Fuchs C. (2017) Social media: a critical introduction. 2nd ed. Ainsley M, ed. London, United Kingdom: SAGE Publications Ltd.

3. Fisher TD. Adolescents' social network site use, peer appearance-related feedback, and body dissatisfaction: testing a mediation model. Sex Roles 2016; 45:211-224.

4. Tiggemann M, Slater A. Facebook and body image concern in adolescent girls: a prospective study. International Journal of Eating Disorders 2017; 50:80-83.

5. Mabe AG, Forney KJ, Keel PK. Do you "like" my photo? Facebook use maintains eating disorder risk. International Journal of Eating Disorders 2014; 47:516-523.

6. Saffran K, Fitzsimmons-Craft EE, Kass AE, et al. Facebook usage among those who have received treatment for an eating disorder in a group setting. International Journal of Eating Disorders 2016; 49:764-777.

7. Fardouly J, Vartanian LR. Negative comparisons about one's appearance mediate the relationship between Facebook usage and body image concerns. Body Image 2015; 12:82-88.

8. Fardouly J, Diedrichs PC, Vartanian LR, et al. The mediating role of appearance comparisons in the relationship between media usage and self-objectification in young women. Psychology of Women Quarterly 2015; 39:447-457.

9. Fox J, Rooney MC. The Dark Triad and trait self-objectification as predictors of men's use and self-presentation behaviors on social networking sites. Personality and Individual Differences 2015; 76:161-165.

10. Kim JW, Chock TM. Body image 2.0: associations between social grooming on Facebook and body image concerns. Computers in Human Behavior 2015; 48:331-339.

11. Brown Z, Tiggemann M. Attractive celebrity and peer images on Instagram: effect on women's mood and body image. Body Image 2016; 19:37-43.

12. Knobloch-Westerwick S. Thinspiration: self-improvement versus self-evaluation social comparisons with thin-ideal media portrayals. Health Communication 2014; 30:10891101.

13. Boepple L, Thompson JK. A content analytic comparison of fitspiration and thinspiration websites. International Journal of Eating Disorders 2016; 49:98-101.

14. Eckler P, Kalyango Y, Paasch E. Facebook use and negative body image among U.S. college women. Women \& Health 2016; 57:249-267.

15. Strubel J, Petrie TA. Love me Tinder: body image and psychosocial functioning among men and women. Body Image 2017; 21:34-38.

16. Fardouly J, Vartanian LR. Cocial media and body image concerns: current research and future directions. Current Opinion in Psychology 2016; 9:1-5.

17. Frederick D, Essayli JH. Male body image: the roles of sexual orientation and body mass index across five national U.S. studies. Psychology of Men \& Masculinity 2016; 17: 336-351. 
18. Carlat DJ, Camargo CA, Herzog DB. Eating disorders in males: a report on 135 patients. American Journal of Psychiatry $1997 ; 154: 1127-1132$.

19. Feldman MB, Meyer IH. Eating disorders in diverse lesbian, gay, and bisexual populations. International Journal of Eating Disorders 2007; 40:218-226.

20. Boroughs MS, Krawczyk R, Thompson JK. Body dysmorphic disorder among diverse racial/ethnic and sexual orientation groups: prevalence estimates and associated factors. Sex Roles 2010; 63:725-737.

21. Schneider SC, Mond J, Turner CM, et al. Sex differences in the presentation of body dysmorphic disorder in a community sample of adolescents. Journal of Clinical Child \& Adolescent Psychology 2017; 5:1-13.

22. Martínez MPL, Berrozpe TI, Lasarte MP. Image-focused social media for a market analysis of tourism consumption. International Journal of Technology Management 2014; 64:17.

23. Goedel W, Brooks F, Duncan D. Approaches to sampling gay, bisexual, and other men who have sex with men from geosocial-networking smartphone applications: a methodological note. Social Sciences 2016; 5:51.

24. Hughto JM, Pachankis JE, Eldahan AI, et al. "You can't just walk down the street and meet someone": the intersection of social-sexual networking technology, stigma, and health among gay and bisexual men in the small city. American Journal of Men's Health 2016; 11:726-736.

25. Phillips G, Magnus M, Kuo I, et al. Use of geosocial networking (GSN) mobile phone applications to find men for sex by men who have sex with men (MSM) in Washington, DC. AIDS and Behavior 2014; 18:1630-1637.

26. Labre MP. Adolescent boys and the muscular male body ideal. Journal of Adolescent Health 2002; 30:233-242.

27. Ridgeway RT, Tylka TL. College men's perceptions of ideal body composition and shape. Psychology of Men \& Masculinity 2005; 6:209-220.

28. Tatangelo GL, Ricciardelli LA. A qualitative study of preadolescent boys' and girls' body image: gendered ideals and sociocultural influences. Body Image 2013; 10:591-598.

29. Griffiths S, Murray SB, Touyz S. Disordered eating and the muscular ideal. Journal of Eating Disorders 2013; 1:1-2.

30. Ip EJ, Barnett MJ, Tenerowicz MJ, et al. Women and anabolic steroids: an analysis of a dozen users. Clinical Journal of Sport Medicine 2010; 20:475-481.

31. Pope HG, Kanayama G, Hudson JI. Risk factors for illicit anabolic-androgenic steroid use in male weightlifters: a cross-sectional cohort study. Biological Psychiatry 2012; 71:254-261.

32. Blashill AJ, Calzo JP, Griffiths S, et al. Anabolic steroid misuse among US adolescent boys: disparities by sexual orientation and race/ethnicity. American Journal of Public Health 2017; 107:319-321.

33. Blashill AJ, Safren SA. Sexual orientation and anabolicandrogenic steroids in US adolescent boys. Pediatrics 2014; 133:469-475.

34. Tiggemann M, Martins Y, Churchett L. Beyond muscles: unexplored parts of men's body image. Journal of Health Psycholgy 2008; 13:1163-1172.

35. Griffiths S, Murray SB, Medeiros A, et al. The tall and the short of it: an investigation of height ideals, height preferences, height dissatisfaction, heightism, and height- related quality of life impairment among sexual minority men. Body Image 2017; 23:146-154.

36. Calzo JP, Blashill AJ, Brown TA, et al. Eating disorders and disordered weight and shape control behaviors in sexual minority populations. Current Psychiatry Reports 2017; 19:225.

37. Kinsey AC, Pomeroy WB, Martin CE. (1948) Sexual behavior in the human male. Indiana: Indiana University Press.

38. Australian Bureau of Statistics. (2016) Australian standard classification of cultural and ethnic groups. Canberra, Australia: Federal Government of Australia.

39. Ryan TA, Morrison TG, Roddy S, et al. Psychometric properties of the Revised Male Body Attitudes Scale among Irish men. Body Image 2011; 8:64-69.

40. Tylka TL, Bergeron D, Schwartz JP. Development and psychometric evaluation of the Male Body Attitudes Scale (MBAS). Body Image 2005; 2:161-175.

41. Gideon N, Hawkes N, Mond J, et al. Development and psychometric validation of the EDE-QS, a 12 item short form of the Eating Disorder Examination Questionnaire (EDE-Q). PLoS One 2016; 11:e0152744.

42. Fairburn C, Beglin S. Assessment of eating disorders: interview or self-report questionnaire? International Journal of Eating Disorders 1994; 16:363-370.

43. Kanayama G, Boynes M, Hudson JI, et al. Anabolic steroid abuse among teenage girls: an illusory problem? Drug and Alcohol Dependence 2007; 88:156-162.

44. Griffiths S, Henshaw R, McKay FH, et al. Post-cycle therapy for performance and image enhancing drug users: a qualitative investigation. Performance Enhancement \& Health 2017; 5:103-107.

45. Hanna E, Ward LM, Seabrook RC, et al. Contributions of social comparison and self-objectification in mediating associations between facebook use and emergent adults' psychological well-being. Cyberpsychology, Behavior, and Social Networking 2017; 20:172-179.

46. Manago AM, Ward LM, Lemm KM, et al. Facebook involvement, objectified body consciousness, body shame, and sexual assertiveness in college women and men. Sex Roles 2015; 72:1-14.

47. McLean SA, Paxton SJ, Wertheim EH, et al. Photoshopping the selfie: self photo editing and photo investment are associated with body dissatisfaction in adolescent girls. International Journal of Eating Disorders 2015; 48:11321140 .

48. Meier EP, Gray J. Facebook photo activity associated with body image disturbance in adolescent girls. Cyberpsychology, Behavior, and Social Networking 2014; 17:199_ 206.

Address correspondence to: Dr. Scott Griffiths Melbourne School of Psychological Sciences University of Melbourne Melbourne VIC 3052 Australia

E-mail: scottgriffiths@gmail.com; scott.griffiths@unimelb.edu.au 